\title{
Monozygotic twinning following embryo biopsy at the blastocyst stage
}

\author{
Rafael Sellers ${ }^{1}$, Juan Carlos Castillo ${ }^{1}$, Jorge Ten ${ }^{1}$, Adoración Rodríguez ${ }^{1}$, José A. Ortiz ${ }^{1}$, Francisco Sellers ${ }^{1}$, \\ Joaquín Llácer ${ }^{1}$, Rafael Bernabeu ${ }^{1}$
}

${ }^{1}$ Reproductive Medicine. Instituto Bernabeu, Alicante, Spain

\begin{abstract}
Objective: Monozygotic twinning incidence following preimplantation genetic testing in embryos at cleavage-stage does not appear to increase; however, data regarding the possible impact of the blastocyst-stage preimplantation genetic testing is lacking. We compared the incidence of monozygotic twinning in preimplantation genetic testing cycles performed at the blastocyst-stage, versus cycles without PGT, following single embryo transfer.

Methods: In this retrospective cohort study, we analyzed the incidence of twin pregnancies in patients undergoing intracytoplasmic sperm injection and blastocyst-preimplantation genetic testing ( 253 cycles), versus a period-matched control population of patients undergoing intracytoplasmic sperm injection and single embryo transfer without preimplantation genetic testing (606 cycles).

Results: The overall monozygotic twinning rate was $14 / 859(1.6 \%)$ per clinical pregnancy. The incidence of zygotic splitting following intracytoplasmic sperm injection and preimplantation genetic testing was 3.5\% (95\% Confidence interval $1.8 \%-6.6 \%$ ) versus $0.8 \%$ (95\% Confidence interval $0.3 \%-1.9 \%$ ) following intracytoplasmic sperm injection without preimplantation sperm injection. After adjusting for potential confounders, preimplantation genetic testing cycles were associated with an increase in the incidence of monozygotic twinning when compared to cycles without embryo biopsy (Odd ratio 3.44, 95\% Confidence interval 1.05-11.27, $p=0.041$ ).

Conclusions: Our findings indicate that embryo biopsy for preimplantation genetic testing performed at the blastocyst stage is associated to an increase in the incidence of monozygotic twinning. Further validation in larger sample size studies is warranted. Patients undergoing preimplantation genetic testing must receive proper counselling about the potential risks of the technique.
\end{abstract}

Keywords: monozygotic twinning, embryo biopsy, blastocyst, preimplantation genetic testing

\section{INTRODUCTION}

Monozygotic twinning (MZT) (the splitting of a single fertilized oocyte into two or more fetuses) occurs in $0.42 \%$ of spontaneous pregnancies (Bulmer, 1970). Monozygotic twin pregnancies are associated with an increased risk of maternal and fetal complications, such as fetal growth restriction, preterm delivery and perinatal mortality (Derom et al., 1987; Malone, 2003). The exact origin of MZT in spontaneous pregnancies remains largely unknown and with the advent of assisted reproduction techniques (ART), several publications have raised concerns regarding the potential for an increased rate of MZT following ART (Abusheikha et al., 2000; Schachter et al., 2001).
Recently, $1.5 \%$ monozygotic twin live birth rate following ART was reported (Mateizel et al., 2016), which appears concordant with the results from a large registered ART data analysis reporting a prevalence of $1.36 \%$ of multiple pregnancies with zygotic splitting even after single embryo transfer (Ikemoto et al., 2018). Additional publications have investigated different ART parameters and their putative involvement in embryo splitting and their potential association with MZT. This includes: maternal age (Ikemoto et al., 2018), ovulation induction (Derom et al., 1987), embryo culture conditions (Edwards et al., 1986), changes to the zona pellucida (ZP) from ICSI and/or assisted hatching (AH) (Hershlag et al., 1999), cryopreservation and prolonged embryo culture up to blastocyst stage (Nakasuji et al., 2014). Taken all together, the body of available evidence suggests an (almost uniform) association between blastocyst transfer and a potential risk of increased MZT; whereas frozen embryo transfer (FET) appears associated with a lower MZT rate. Other ART parameters appear equivocal and warrant future studies (Mateizel et al., 2016; Ikemoto et al., 2018).

Recently, following a revision in ART terminology (Zegers-Hochschild et al., 2017), the term "preimplantation genetic testing" (PGT) was introduced, to describe the test performed to analyze the DNA from oocytes (polar bodies) or embryos (cleavage stage or blastocyst) for HLA typing or for determining genetic abnormalities. Publications on the incidence of MZT after the use of PGT in ART cycles are scarce. At least theoretically, the incidence of MZT in PGT cycles must be potentially increased because of different mechanisms, including: intentional holes in the ZP and cell manipulation for biopsy. To the best of our knowledge, the only available publication on the topic reported that the incidence of MZT did not increase in PGT cycles compared with regular intracytoplasmic sperm injection with blastocyst transfer cycles (Verpoest et al., 2009), this publication included cleavage-stage embryo biopsies exclusively. More recently, cleavage-stage embryo biopsies have been largely replaced by trophectoderm (TE) biopsies. Blastocyst biopsy provides more cells and is performed at an embryonic stage more amenable to genetic analyses, and less sensitive to possible damage (Coll et al., 2018). We lack reports on the incidence of MZT after the use of ART in combination with PGT performed at the blastocyst stage.

The aim of this study was to assess the incidence of MZT pregnancies in a cohort of consecutive PGT treatment cycles performed at the blastocyst stage, followed by single blastocyst embryo transfer, in comparison with a period-matched cohort of consecutive ICSI cycles with single embryo transfer (SET) at the same center.

\section{MATERIAL AND METHODS}

In this retrospective comparative cohort study, we analyzed the incidence of MZT pregnancies in a cohort of consecutive ICSI treatment cycles combined with PGT by TE 
biopsy and single blastocyst embryo transfer (study group) and compared the results with a control cohort of consecutive ICSI cycles and SET without embryo biopsy (control group). The study was conducted at a single private center for reproductive medicine and included data from January 2014 to December 2017.

\section{Study and control groups}

ART cycles included own-eggs and donor-eggs cycles. Specific characteristics of the ovarian stimulation process were not included for analysis but there were no differences in laboratory conditions vis-à-vis procedures between control and study groups, except for the AH (on day-3 of embryo development) and TE biopsy procedure for PGT in the study group. Once collected, the oocytes were incubated in a culture medium (Global Total for Fertilization, LifeGlobal ${ }^{\circledR}$ ) until denudation for subsequent ICSI (usually two hours later). In the study group, ICSI was performed in order to prevent contamination with residual sperm DNA (Liebaers et al., 1998). The details of the ICSI procedure have been described previously (Lledó et al., 2006). Fertilization was assessed 16-18 hours after ICSI and the embryos obtained remained in one-step culture medium (Global Total, LifeGlobal ${ }^{\circledR}$ ). Further development was evaluated in the morning of day 3 when laser-AH (Lykos ${ }^{\circledR}$, Hamilton Thorne ${ }^{\circledR}$ ) was performed as previously described (Lledó et al., 2006). At the blastocyst stage, the embryos were classified according to morphologic characteristics by ASEBIR (2015). Laser-assisted biopsy and aspiration was applied consistently to remove five to eight TE cells.

Within the study group, depending on clinical decision, biopsied embryos were either selected for fresh transfer the day after biopsy (study-fresh group), vitrifying supernumerary euploid embryos, or for elective complete vitrification (study-frozen group) and deferred embryo transfer. Genetic analysis was performed using array-CGH (which provides results within 24 hours) in the fresh group, whereas Next Generation Sequencing (NGS) was used in the frozen group. Similarly, in the control group and depending upon clinical decision, embryos were selected either for fresh transfer (control-fresh group) plus vitrification of surplus embryos or for elective complete vitrification (control-frozen group) for deferred embryo transfer. A single embryo transfer was performed in all cases; cleavage or blastocyst stage transfer was allowed in the control group.

Endometrial preparation in frozen-thawed embryo transfer (FET) artificial cycles (exogenous estrogen administration plus timely addition of progesterone) as well as natural cycle endometrial preparation used as per clinical decision. Specific characteristics of the endometrial preparation process were not included in the analysis.

\section{MZT diagnosis}

A clinical pregnancy was defined as one or more gestational sacs seen via transvaginal ultrasound scan at least 5 weeks after embryo transfer. MZT was identified at first ultrasound when the number of fetal heartbeats exceeded the number of gestational sacs, or when the number of sacs exceeded the number of embryos transferred.

\section{Statistical analysis}

For the statistical analysis of patients and cycle characteristics, continuous variables were presented as number of cases, mean and typical deviation. A $p=0.05$ was considered statistically significant after performing a t-student test. Categorical variables were presented as number of cases, percentage and odds ratio (OR). Pearson's Chi-square test (univariate) and binary logistic regression (multivariate for confounding factors) were used to analyze association of specific ART features and MZT. A $p$-value was considered significant if $<0.05$. The statistical analysis was performed using the SPSS 20.0 software (SPSS, Chicago, IL, USA).

\section{RESULTS}

Overall, we had 859 clinical pregnancies after SET during the study period. A total 606 cycles corresponded to ICSI and single embryo transfer without PGT and 253 cycles to patients undergoing ICSI and PGT (for various indications) with single blastocyst transfer. Of these, 14 resulted in twin pregnancies (global MZT rate $1.6 \%$ per clinical pregnancy). Globally, $93.3 \%$ of the cycles included embryo transfer at the blastocyst stage. Table 1 depicts the distribution of the baseline cycle characteristics within the MZT and singleton groups. Monozygotic splitting occurred significantly more frequently $(p=0.004)$ in PGT cycles. Frozen embryo transfer cycles were found more frequently associated with MZT without reaching statistical significance $(p=0.06)$.

Table 2 shows that the incidence of zygotic splitting following PGT was $3.5 \%$ (95\% CI $1.8 \%$ - $6.6 \%$ ) versus $0.8 \%(95 \%$ CI $0.3 \%-1.9 \%)$ following ICSI (without embryo biopsy).

Table 3 presents a logistic regression analysis evaluating the effect of age and specific cycle parameters over the incidence of MZT. In the univariate analysis, cycles using donated eggs were more associated with a significant increased risk in monozygotic splitting when compared to own-eggs ICSI cycles (OR 5.01, 95\% CI 1.1 - 22.69). The transfer of a frozen embryo also resulted in a significantly increased risk for MZT when compared to a fresh embryo in the univariate analysis (OR 4.12, 95\% CI 1.04 - 16.18). After adjusting for these potential confounders (source of eggs and fresh/frozen cycles), the PGT procedure was associated with a significant increase in the incidence of MZT when compared to a non-biopsied embryo (OR 3.44, 95\% CI 1.05-11.27, $p=0.041$ ).

Table 4 shows a sub-analysis considering blastocyst-stage transfers only in the control group. The frequency of monozygotic splitting remains statistically significant $(p=0.006)$ in PGT cycles as occurred in the overall analysis (Table 1). However, after adjusting for the described potential confounders (source of the eggs and type of ET cycle), the PGT group was more frequently associated with MZT, but without reaching statistical significance $(p=0.066)$.

\section{DISCUSSION}

To the best of our knowledge, this is the first study focused on exploring the potential effect of the embryo biopsy procedure at the blastocyst stage vis-a-vis the incidence of monozygotic twin gestations. Notwithstanding the weaknesses associated to its retrospective design (including residual confounding) and limited sample, still our findings can contribute to the body of (scarce) medical evidence on the subject. We report an increased risk of monozygotic splitting with the PGT embryo biopsy procedure when performed at blastocyst stage in ART cycles.

The main strength of our study is the inclusion of cycles receiving only a single embryo for transfer and a vast proportion of embryos transferred at the blastocyst stage, thereby limiting the potential bias associated with the transfer of multiple embryos at different embryonic stages.

Even after single embryo transfer, we had a $1.6 \%$ monozygotic twin rate, higher than what is reported after spontaneous conception (0.40-0.45\%; Aston et al., 2008), but similar to previous reports in ART cycles $(1.4 \%, \mathrm{Na}-$ kasuji et al., 2014); 2.2\%, Mateizel et al., 2016) including PGT only cycles (1.9\%, Verpoest et al., 2009). Even though the absolute numbers are quite small, these findings additionally confirm that ART constitutes a risk factor for monozygotic pregnancies. 
Table 1. Age and specific cycle characteristics and their incidence in singleton and monozygotic splitting groups

\begin{tabular}{|l|c|c|c|}
\hline Age and specific cycle characteristics & $\begin{array}{c}\text { Singleton } \\
(\mathbf{n = 8 4 5 )}\end{array}$ & $\begin{array}{c}\text { MZT } \\
(\mathbf{n = 1 4 )}\end{array}$ & $\boldsymbol{p}$-value \\
\hline Age Mean \pm SD & $39.93 \pm 4.96$ & $38.42 \pm 5.27$ & $0.295^{\mathrm{a}}$ \\
Maternal & $24.77 \pm 3.74$ & $23.50 \pm 4.95$ & $0.632^{\mathrm{a}}$ \\
Donor & $41.74 \pm 7.08$ & $38.67 \pm 4.04$ & $0.452^{\mathrm{a}}$ \\
Paternal & $273(32.3)$ & $6(42.9)$ & $0.403^{\mathrm{b}}$ \\
\hline Source of the eggs $\mathrm{n}(\%)$ & $572(67.7)$ & $8(57.1)$ & \\
Own & & $4(28.6)$ & $0.060^{\mathrm{b}}$ \\
Donated & $455(53.8)$ & $10(71.4)$ & \\
\hline Type of ET cycle $\mathrm{n}(\%)$ & $390(46.2)$ & $5(35.7)$ & $9.004^{\mathrm{b}}$ \\
Fresh & $601(71.1)$ & $9(64.3)$ & \\
\hline Prozen & $244(28.9)$ & & \\
\hline
\end{tabular}

aStudent's test

'Pearson's chi-squared test $\left(X^{2}\right)$

\begin{tabular}{|l|c|}
\hline \multicolumn{2}{|l|}{ Table 2. Risk MZT } \\
\hline PGT-A n (\%) & MTZ Risk (95\% IC) \\
\hline- & $0.825(0.353-1.917)$ \\
\hline+ & $3.557(1.883-6.621)$ \\
\hline
\end{tabular}

Table 3. The association between ART parameters and MZT

\begin{tabular}{|l|c|c|}
\hline ART parameters & OR (95\% CI) & p-value \\
\hline Source of the eggs & $4.121(0.436-13.450)$ & $0.312^{\mathrm{a}}$ \\
Own & $5.011(1.107-22-694)$ & $0.036^{\mathrm{a}}$ \\
Donated & & \\
\hline Type of ET cycle & $3.417(0.476-24.560)$ & $0.222^{\mathrm{a}}$ \\
Fresh & $4.121(1.049-16.187)$ & $0.043^{\mathrm{a}}$ \\
\hline Frozen & $3.448(1.054-11.279)$ & $0.041^{\mathrm{b}}$ \\
\hline Global
\end{tabular}

anivariate logistic regression

${ }^{b}$ Adjusted multivariate logistic regression. Confounding factors: Source of the eggs and type of ET cycle

Table 4. Incidence in singleton and monozygotic splitting groups only on blastocyst-stage transfers $(D+5 / D+6)$

\begin{tabular}{|c|c|c|c|}
\hline \multirow[t]{2}{*}{ blastocyst } & Singleton & MZT & p-value \\
\hline & $(n=775)$ & $(n=14)$ & \\
\hline $\begin{array}{l}\text { PGT-A n (\%) } \\
- \\
+\end{array}$ & $\begin{array}{l}536(69.2) \\
239(30.8)\end{array}$ & $\begin{array}{l}5(35.7) \\
9(64.3)\end{array}$ & $0.006^{a}$ \\
\hline Global OR (95\% CI) & \multicolumn{2}{|c|}{$3.113(0.928-10.443)$} & $0.066^{b}$ \\
\hline
\end{tabular}

aPearson's chi-squared test $\left(X^{2}\right)$

${ }^{b}$ Adjusted multivariate logistic regression. Confounding factors: Source of the eggs and type of ET cycle
More specifically, within these SET cycles only, we considered exploring for certain cycle parameters and their possible association with embryo splitting; the source of the eggs: own vs donated (proxy for the potential impact of age), fresh vs frozen embryo transfer cycle and TE biopsy for PGT. According to our data, donor-eggs appear to be associated with an increased risk of MZT. This finding is concordant with a previous publication (Luke et al., 2014), but in disagreement with a previous meta-analysis finding no association between the use of donor-eggs and the risk of MZT (Busnelli et al., 2019), among the possible explanations for these discrepancies are the number and stage of the embryos transferred from oocyte-donors in other studies which (together with other residual confounders) may introduce a source of bias. Interestingly, in the case of own-eggs cycles, the meta-analysis by Busnelli et al. (2019) found that embryos derived from younger oocytes (i.e., female age at time of retrieval $<35$ years) were significantly more likely to result in an MZT pregnancy. Nonetheless, blastocyst transfer was considered as a potential confounding factor, since it is well known that embryos derived from young oocytes are more likely to be transferred at an advanced blastocyst stage, finally concluding that age may not be an independent risk factor for MZT but rather a proxy for blastocyst transfer (Busnelli et al., 2019). However, in our study, the vast majority of embryos $(93.3 \%)$ were transferred at the blastocyst stage and still donor-eggs remained associated to an increased risk for monozygotic splitting. As an additional note, one previous study has reported that two MZT resulted from the transfer of embryos derived from a single oocyte donor's retrieval and also another oocyte recipient who underwent a two-ET conceived quadruplets (Knopman et al., 2014). Further data is warranted to deepen in the exact etiology behind the association of younger age and MZT, beyond the (probwably) valid but unspecific: "healthier oocytes and superior reproductive potential" argument (Knopman et al., 2014).

MZT is a low rate event; thus, a high number of cases should be analyzed to find significant differences. In terms of embryo stage upon ET, since the blastocyst stage embryo transfer is highly predominant in our center, we 
expected a vast majority of the control cases to fit into the blastocyst stage transfer category. Indeed, the vast proportion of transfers in the control group (93.3\%) were in the blastocyst stage. To expand on the issue, we performed statistics including blastocyst-stage transfers only day-5/day-6 $(n=775)$, which resulted in similar outcomes in the crude analyses, consistently showing an increase in the MZT rate associated with the embryo biopsy procedure (Table 4). Nonetheless, -and presumably as a consequence of the reduction in sample size-, the significance is lost when accounting for confounders in the data analysis, even though a trend is evident. Taken all together, even acknowledging the absence of statistical significance in the blastocyst-only sub-analysis, our data is still concordant with an increased risk for MZT associated with the embryo biopsy procedure.

Regarding fresh vs. frozen cycles, our study found that the transfer of a frozen embryo was associated with an increased risk of MZT in the univariate analysis. The same finding has been reported by several (Alikani et al., 2003; Knopman et al., 2014; Nakasuji et al., 2014; Ikemoto et al., 2018) but not all previous publications (Mateizel et al., 2016; Busnelli et al., 2019) on the topic. It is worth noting that a significant proportion of PGT cycles in our study were transferred in a frozen cycle $(148 / 253,58.5 \%)$. Hence, the possibility exists that the frozen cycle may act as a confounding factor. Consequently, frozen cycles might not be a true independent risk factor for MZT, but rather a proxy for PGT impact. An important limitation of our observational study is that data regarding frozen-warmed embryo transfer cycles did not include information about specific endometrial preparation protocols, which may have influenced the outcome (e.g., an endometrial preparation in a natural cycle may have contributed to an "additional" natural conception). Therefore, a causal/casual relationship between zygotic splitting and embryo-cryopreservation-frozen/thaw ART procedures might be suggested, but cannot be demonstrated from our data.

Embryo PGT entails several invasive procedures and manipulations including breaks, (laser-assisted) intentional holes in the ZP and TE cells biopsy, all of which could potentially increase MZT rates (Verpoest et al., 2009). According to our data, even after adjusting for potential confounders, PGT cycles remain a significant factor associated with an increased risk for monozygotic splitting compared to ICSI cycles. Our findings contradict a previously reported data on the topic, which showed no increase in MZT rate and PGT-A compared to regular ICSI after fresh blastocyst transfer (Verpoest et al., 2009). This divergence may be attributed to several factors. First, the number of embryos for transfer. In the study by Verpoest et al. (2009), a mean of 1.6 embryos (in each arm) were transferred and, -as acknowledged by authors-, chorionicity was not clearly established in MZT pregnancies. This policy may have introduced a substantial bias since a causal relationship between the higher number of embryos transferred and MZT has been argued (Sills et al., 2000). In our study, albeit chorionicity or DNA fingerprinting were also not addressed, the inclusion of SET only cycles would have limited this potential source of bias. Secondly, the inclusion of fresh-only cycles by Verpoest et al. (2009); as previously discussed, almost $60 \%$ of PGT cycles in our study included frozen-thawed cycles, which may have additionally contributed to the MZT outcome as suggested in the univariate analysis. Specific changes in the ZP or TE cells during/after vitrification in a blastocyst-stage biopsied embryo could be hypothesized as potential additional factors associated with the increased risk of MZT in frozen cycles.

This hypothesis warrants further investigations. Third, and of the utmost importance, the difference in the embryo-stage when performing the biopsy. The study by Verpoest et al. (2009) included cleavage-stage embryo biopsies, which is a less invasive approach if compared with the TE blastocyst stage embryo biopsy. Additional to the procedures for cleavage-stage biopsies, blastocyst biopsies add aspiration and laser-assisted excision in combination with mechanical removal of TE cells by "pulling" or "flicking". A possible factor explaining the split of the inner mass cell (IMC) during long culture up to the blastocyst stage for subsequent embryo biopsy is the exposure of the embryo to low concentrations of $\mathrm{Ca}^{+2}$ in the culture media. These low concentrations may promote less cells-adhesion and a "weakening" of the inter-cellular junctions provoking the detachment of some cells from the IMC and the formation of an extra embryonic pole in the TE (Milki et al., 2003). Therefore, it can be hypothesized that the exposure of the embryo to low $\mathrm{Ca}^{+2}$ concentrations together with the mechanical manipulation during embryo biopsy may act as added factors (chemical and mechanical) favoring a later splitting of the ICM, resulting in MZT. While some reports may support this hypothesis (Edwards et al., 1986; Van Langendonckt et al., 2000; Yan et al., 2015), a recent retrospective evaluation focused on PGT cycles (Gu et al., 2018) taking into account the "hatching" status of the blastocyst (partially and fully hatched blastocysts vs 8-shaped blastocysts with ICM incarceration) before biopsy and the relation with embryo splitting, showed that ICM incarceration in 8-shaped blastocysts did not increase MZT incidence. While some differences in the protocol may explain this discrepancy, Gu et al. (2018) still reported an overall MZT pregnancy rate per established clinical pregnancy of $2.8 \%$ (similar to our rate). Unfortunately, the study did not include regular ICSI with blastocyst transfer for comparison. In concordance with our study, the (additional) influence of $\mathrm{AH}$ in the MZT rate could not be disentangled in the study by $\mathrm{Gu}$ et al. (2018), because all the embryos in the PGT group received $\mathrm{AH}$ on day 3 of embryo development. But, as suggested by some observations, even if the zona manipulation on cleavage-stage (i.e., $\mathrm{AH}$ ) may be involved in some cases of MZT, it is unlikely to be an exclusive mechanism ( $\mathrm{Gu}$ et al., 2018). Nonetheless, in the study by Verpoest et al. (2009) cleavage stage embryo-biopsies did not show correlation with an increase in MZT rate; in these regards, cleavage-stage biopsy could be inferred as a proxy for $\mathrm{AH}$ (both procedures involves "making a hole" in the zona pellucida during cleavage stage).

Also, some additional drawbacks from our study merits further analysis. MZT is - fortunately- an infrequent event; indeed studies focused on rare phenomenon constitute a challenge. Obviously, the results from our study, with a limited sample size, should be further explored in large databases in order to reach robust conclusions. However, due to the sharp increase in PGT cycles worldwide, even small increases in incidences may translate into important crude numbers of this potentially complex obstetric condition. The results from our study may inspire future studies on the subject. The lack of confirmation of monozygocity at birth constitutes another arguable limitation of the study; we believe that this approach is only of marginal importance, and it is not crucial for the purpose of diagnosis of am MZT (especially after SET policy); several publications on the topic have clearly established that transvaginal ultrasound is highly accurate in diagnosing a MZT pregnancy; moreover all cases in our study received one additional and dedicated evaluation of the MZT status on the week after the initial visualization for confirmation (weeks 6-7). Notwithstanding these potential limitations, still the results found in our study can contribute to the body of medical evidence on the subject.

\section{CONCLUSION}

Our findings indicate that embryo biopsy for PGT performed at the blastocyst stage is associated to an increase in the incidence of MZT. Because of the increased 
risk associated with MZT, this information could be useful to help identify potential factors underlying the higher incidence of MZT in the context of ART, and to identify strategies to reduce this incidence. Given the low event rates with monozygotic twinning, our results warrant validation in larger sample size studies required to provide higher statistical power. Until those studies become available, patients undergoing PGT must receive proper counselling about the potential risks of the technique.

\section{CONFLICT OF INTEREST}

The authors certify that they have NO affiliations with or involvement in any organization or entity with any financial interest (such as honoraria; educational grants; participation in speakers' bureaus; membership, employment, consultancies, stock ownership, or other equity interest; and expert testimony or patent-licensing arrangements), or non-financial interest (such as personal or professional relationships, affiliations, knowledge or beliefs) in the subject matter or materials discussed in this manuscript. No external funding was sought or obtained for this study

\section{Corresponding Author:}

Juan Carlos Castillo

Reproductive Medicine

Instituto Bernabeu

Alicante, Spain.

E-mail: jcastillo@institutobernabeu.com

\section{REFERENCES}

Abusheikha N, Salha O, Sharma V, Brinsden P. Monozygotic twinning and IVF/ICSI treatment: a report of 11 cases and review of literature. Hum Reprod Update. 2000;6:396403. PMID: 10972526 DOI: 10.1093/humupd/6.4.396

Alikani M, Cekleniak NA, Walters E, Cohen J. Monozygotic twinning following assisted conception: an analysis of 81 consecutive cases. Hum Reprod. 2003;18:1937-43. PMID: 12923153 DOI: 10.1093/humrep/deg369

ASEBIR - Asociación para el Estudio de la Biologia de la Reproduction. Criterios ASEBIR de Valoración Morfológica de Oocitos, Embriones Tempranos y Blastocistos Humanos. Cuadernos de Embriología Clínica [Internet]; 2015. Available at: https://asebir.com/cuadernos-asebir/ criterios-asebir-de-valoracion-morfologica-de-oocitos-embriones-tempranos-y-blastocistos-humanos/

Aston KI, Peterson CM, Carrell DT. Monozygotic twinning associated with assisted reproductive technologies: a review. Reproduction. 2008;136:377-86. PMID: 18577552 DOI: $10.1530 /$ REP-08-0206

Bulmer MG. The biology of twinning in man. Oxford: Clarendon Press; 1970.

Busnelli A, Dallagiovanna C, Reschini M, Paffoni A, Fedele L, Somigliana E. Risk factors for monozygotic twinning after in vitro fertilization: a systematic review and meta-analysis. Fertil Steril. 2019;111:302-17. PMID: 30691632 DOI: 10.1016/j.fertnstert.2018.10.025

Coll L, Parriego M, Boada M, Devesa M, Arroyo G, Rodríguez I, Coroleu B, Vidal F, Veiga A. Transition from blastomere to trophectoderm biopsy: comparing two preimplantation genetic testing for aneuploidies strategies. Zygote. 2018;26:191-8. PMID: 29798732 DOI: 10.1017/S0967199418000084
Derom C, Vlietinck R, Derom R, Van den Berghe H, Thiery $M$. Increased monozygotic twinning rate after ovulation induction. Lancet. 1987;1:1236-8. PMID: 2884372 DOI: $10.1016 / \mathrm{S} 0140-6736(87) 92688-2$

Edwards RG, Mettler L, Walters DE. Identical twins and in vitro fertilization. J In Vitro Fert Embryo Transf. 1986;3:1147. PMID: 3701181 DOI: 10.1007/BF01139357

Gu YF, Zhou QW, Zhang SP, Lu CF, Gong F, Tan YQ, Lu GX, Lin $\mathrm{G}$. Inner cell mass incarceration in 8-shaped blastocysts does not increase monozygotic twinning in preimplantation genetic diagnosis and screening patients. PLoS One. 2018;13:e0190776. PMID: 29315321 DOI: 10.1371/ journal.pone.0190776

Hershlag A, Paine T, Cooper GW, Scholl GM, Rawlinson K, Kvapil G. Monozygotic twinning associated with mechanical assisted hatching. Fertil Steril. 1999;71:144-6. PMID: 9935131 DOI: 10.1016/S0015-0282(98)00402-6

Ikemoto Y, Kuroda K, Ochiai A, Yamashita S, Ikuma S, Nojiri S, Itakura A, Takeda S. Prevalence and risk factors of zygotic splitting after 937848 single embryo transfer cycles. Hum Reprod. 2018;33:1984-91. PMID: 30299468 DOI: $10.1093 /$ humrep/dey294

Knopman JM, Krey LC, Oh C, Lee J, McCaffrey C, Noyes $\mathrm{N}$. What makes them split? Identifying risk factors that lead to monozygotic twins after in vitro fertilization. Fertil Steril. 2014;102:82-9. PMID: 24794318 DOI: 10.1016/j. fertnstert.2014.03.039

Liebaers I, Sermon K, Staessen C, Joris H, Lissens W, Van Assche E, Nagy P, Bonduelle M, Vandervorst M, Devroey P, Van Steirteghem A. Clinical experience with preimplantation genetic diagnosis and intracytoplasmic sperm injection. Hum Reprod. 1998;13:186-95. PMID: 9663783 DOI: 10.1093/humrep/13.suppl_1.186

Lledó B, Ten J, Galán FM, Bernabeu R. Preimplantation genetic diagnosis of Marfan syndrome using multiple displacement amplification. Fertil Steril. 2006;86:949-55. PMID: 17027361 DOI: 10.1016/j.fertnstert.2006.03.036

Luke B, Brown MB, Wantman E, Stern JE. Factors associated with monozygosity in assisted reproductive technology pregnancies and the risk of recurrence using linked cycles. Fertil Steril. 2014;101:683-9. PMID: 24388206 DOI: 10.1016/j.fertnstert.2013.11.034

Malone FD. Monochorionic pregnancy--where have we been? Where are we going? Am J Obstet Gynecol. 2003;189:1308-9. PMID: 14634559 DOI: 10.1067/j. ajog.2003.10.682

Mateizel I, Santos-Ribeiro S, Done E, Van Landuyt L, Van de Velde $H$, Tournaye $H$, Verheyen $G$. Do ARTs affect the incidence of monozygotic twinning? Hum Reprod. 2016;31:2435-41. PMID: 27664211 DOI: 10.1093/humrep/dew216

Milki AA, Jun SH, Hinckley MD, Behr B, Giudice LC, Westphal LM. Incidence of monozygotic twinning with blastocyst transfer compared to cleavage-stage transfer. Fertil Steril. 2003;79:503-6. PMID: 12620430 DOI: 10.1016/ s0015-0282(02)04754-4 
Nakasuji T, Saito H, Araki R, Nakaza A, Nakashima A, Kuwahara $A$, Ishihara $O$, Irahara $M$, Kubota $T$, Yoshimura $Y$, Sakumoto $\mathrm{T}$. The incidence of monozygotic twinning in assisted reproductive technology: analysis based on results from the 2010 Japanese ART national registry. J Assist Reprod Genet. 2014;31:803-7. PMID: 24722789 DOI: $10.1007 / \mathrm{s} 10815-014-0225-0$

Schachter M, Raziel A, Friedler S, Strassburger D, Bern O, Ron-El R. Monozygotic twinning after assisted reproductive techniques: a phenomenon independent of micromanipulation. Hum Reprod. 2001;16:1264-9. PMID: 11387303 DOI: $10.1093 /$ humrep/16.6.1264

Sills ES, Moomjy M, Zaninovic N, Veeck LL, McGee M, Palermo GD, Rosenwaks Z. Human zona pellucida micromanipulation and monozygotic twinning frequency after IVF. Hum Reprod. 2000;15:890-5. PMID: 10739838 DOI: 10.1093/humrep/15.4.890
Van Langendonckt A, Wyns C, Godin PA, Toussaint-Demylle $D$, Donnez J. Atypical hatching of a human blastocyst leading to monozygotic twinning: a case report. Fertil Steril. 2000;74:1047-50. PMID: 11056259 DOI: 10.1016/S00150282(00)01554-5

Verpoest W, Van Landuyt L, Desmyttere S, Cremers A, Devroey $\mathrm{P}$, Liebaers $\mathrm{I}$. The incidence of monozygotic twinning following PGD is not increased. Hum Reprod. 2009;24:2945-50. PMID: 19661123 DOI: 10.1093/humrep/dep280

Yan $Z$, Liang $H$, Deng $L$, Long $H$, Chen $H$, Chai W, Suo L, Xu C, Kuang Y, Wu L, Lu S, Lyu Q. Eight-Shaped Hatching Increases the Risk of Inner Cell Mass Splitting in Extended Mouse Embryo Culture. PLoS One. 2015;10:e0145172. PMID: 26680631 DOI: 10.1371/journal.pone.0145172

Zegers-Hochschild F, Adamson GD, Dyer S, Racowsky C, de Mouzon J, Sokol R, Rienzi L, Sunde A, Schmidt L, Cooke ID, Simpson JL, van der Poel S. The International Glossary on Infertility and Fertility Care, 2017. Fertil Steril. 2017;108:393406. PMID: 28760517 DOI: 10.1016/j.fertnstert.2017.06.005 